\title{
THE ANTIOXIDANT POTENTIAL EVALUATION OF DICLOFENAC DERIVATIVES WITH HYDRAZONES STRUCTURE
}

\author{
ALIN FOCȘA ${ }^{1}$, ANDREEA IACOB ${ }^{1}$, IOANA VASINCU ${ }^{1}$, SANDRA CONSTANTIN ${ }^{1}$, \\ LOREDANA ANDRIESCU ${ }^{1}$, ALEXANDRU SAVA ${ }^{1}$, FRÉDÉRIC BURON ${ }^{2}$, SYLVAIN ROUTIER $^{2}$, \\ LENUȚA PROFIRE $^{1 *}$ \\ ${ }^{I}$ Department of Pharmaceutical Chemistry, Faculty of Pharmacy, "Grigore T. Popa” University of Medicine and Pharmacy, \\ 16 Universității Street, 700115, Iaşi, Romania \\ ${ }^{2}$ Institute of Organic and Analytical Chemistry, University of Orléans - Pôle de Chimie, Chartres Street, 45067, Orléans, France
}

*corresponding author: lenuta.profire@umfiasi.ro

Manuscript received: October 2019

\begin{abstract}
A series of diclofenac hydrazones was synthesized and the structure of the compounds was proved using different spectral methods such as infrared (IR), nuclear magnetic resonance ( $\left.{ }^{1} \mathrm{H}-\mathrm{NMR}\right)$ and high resolution mass spectroscopy (HR-MS). The biological evaluation, focused on in vitro antioxidant effects using DPPH (2,2-diphenyl-1-picrylhydrazyl) and ABTS (2,2'azino-bis-(3-ethylbenzothiazoline-6-sulfonic acid)) free radicals scavenging assays was also performed. An improved antioxidant effect of the synthesized compounds compared to diclofenac was observed.
\end{abstract}

\section{Rezumat}

O serie de derivați ai diclofenacului cu structură de hidrazonă a fost sintetizată, iar structura compușilor a fost confirmată utilizându-se diferite metode spectrale: spectroscopie în infraroșu (IR), rezonanță magnetică nucleară $\left({ }^{1} \mathrm{H}-\mathrm{RMN}\right)$ și spectroscopie de masă de înaltă rezoluție (HR-MS). Evaluarea biologică a urmărit determinarea in vitro a efectelor antioxidante față de radicalii DPPH (1,1-difenil-2-picrilhidrazil) și ABTS (acid 2,2'-azinobis(3-etilbenzotiazolin-6-sulfonic)). Pentru compuşii sintetizați s-a observat o acțiune antioxidantă îmbunătațită comparativ cu cea a diclofenacului.

Keywords: diclofenac, hydrazone, antioxidant effects

\section{Introduction}

Classical NSAIDs (nonsteroidal anti-inflammatory drugs) are one of the most used class of medication worldwide, primarily based on their effectiveness as anti-inflammatory, analgesic and antipyretic agents $[9,12,15]$. Since diclofenac therapy is associated with several side effects, especially at the gastrointestinal and renal level, the researchers have focused their research work on the derivatization at the free carboxyl group in order to obtain new compounds with improved toxicological profile. Recent studies have also highlighted that reactive oxygen species are involved in triggering of various diseases including inflammatory conditions and the diseases associated [2,5].

Advances in molecular biology and rational drug design approaches have led to the successful identification of novel anti-inflammatory targets $[10,11]$. In order to improve the pharmacological profile and to reduce the side effects of the diclofenac, a series of hydrazones derivatives of diclofenac has been synthetized and biologically evaluated in terms of antioxidant effects.

\section{Materials and Methods}

Reagents: diclofenac sodium, hydrazine hydrate $64 \%$, aromatic aldehydes (2-nitro/4-cyan/3-nitro/3-ethoxy4-hydroxy/4-bromo-2-nitro/2-chloro-5-trifluoromethyl/4-methoxy/4-methyl/3-trifluormethyl/3,4difluoro/3-bromo-4-hydroxy/2,5-dibromo/2-bromo4-fluoro/4-bromo-2-fluoro/3-fluoro-4-methyl/4fluoro/3-methoxy-4-methyl/2-bromo-3-hydroxy-4methoxy - benzaldehyde), solvents proanalysis (p.a.) (ethanol, dioxane, diethyl ether, ethyl acetate, petroleum ether, n-pentane), reagents used as catalysts (acetic acid, sulfuric acid, hydrochloric acid), dimethylsulphoxide- $\mathrm{d}_{6}\left(\right.$ DMSO- $\left.\mathrm{d}_{6}\right)$, standard reagents for radical scavenging assays were purchased from Sigma Aldrich. All reagents and solvents were used without prior purification.

Synthesis. The synthesis of hydrazone derivatives of diclofenac was performed according to the procedure described in Figure $1[3,6]$.

The reactions were monitored using TLC (Thin Layer Chromatography) Silica gel 60 F254 plates produced by Merck Company and the spots were visualized using UV light. 

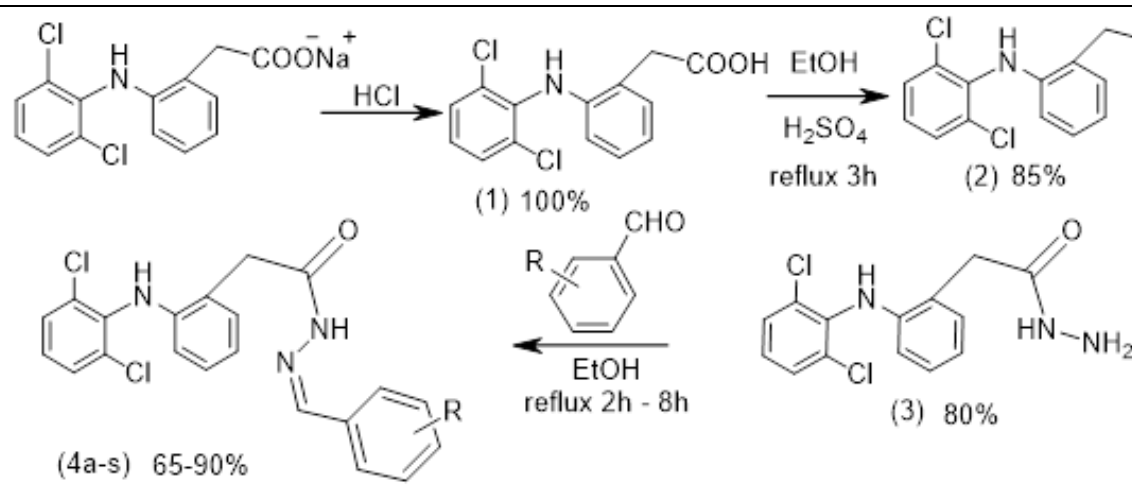<smiles>CCOCCCO</smiles>

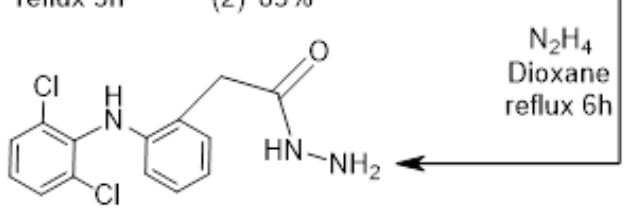

(3) $80 \%$

Figure 1.

The synthesis of hydrazone derivatives of diclofenac (4a-s)

Physico-chemical and spectral characterization. The melting points were measured using a Buchi Melting Point B-540 apparatus and they are uncorrected. The infrared (IR) spectra were recorded on a ThermoNicolet AVATAR 320 AEK0200713 FT-IR Spectrometer, at a resolution of $4 \mathrm{~cm}^{-1}$ after 6 scans in the $4000-500 \mathrm{~cm}^{-1}$ range. The spectra were processed using the Omnic Spectra Software. The ${ }^{1} \mathrm{H}-\mathrm{NMR}$ spectra were recorded with a Bruker Avance Spectrometer $400 \mathrm{MHz}$, using tetramethylsilane (TMS) as internal standard and DMSO- $\mathrm{d}_{6}$ as solvent. The chemical shifts were shown in $\delta$ values (ppm). The mass spectra were recorded using a Bruker MaXis Ultra-High Resolution Quadrupole Time-of-Flight Mass Spectrometer.

Biological evaluation. The antioxidant effects were evaluated using DPPH (2,2-diphenyl-1-picrylhydrazyl) and ABTS (2,2'-azino-bis-(3-ethylbenzothiazoline6-sulfonic acid)) free radicals scavenging assays.

The DPPH Radical Scavenging Assay. The tested compounds were dissolved in DMSO to obtain a stock solution of $10 \mathrm{mg} / \mathrm{mL}$. From the stock solution there were taken different samples $(50 \mu \mathrm{L}, 100 \mu \mathrm{L}, 200 \mu \mathrm{L}$, $300 \mu \mathrm{L}, 400 \mu \mathrm{L}, 500 \mu \mathrm{L}$ ) which were diluted with methanol in order to obtain $1300 \mu \mathrm{L}$. Over the resulting samples $1200 \mu \mathrm{L}$ of methanol solution of DPPH $(0.1 \mathrm{mM}$, $\left.\mathrm{A}_{517 \mathrm{~nm}}=1.0 \pm 0.05\right)$ were added. The concentration of the compounds tested in the obtained samples was $0.2 \mathrm{mg} / \mathrm{mL}, 0.4 \mathrm{mg} / \mathrm{mL}, 0.8 \mathrm{mg} / \mathrm{mL}, 1.2 \mathrm{mg} / \mathrm{mL}$, $1.6 \mathrm{mg} / \mathrm{mL}$ and $2.0 \mathrm{mg} / \mathrm{mL}$ respectively. The mixture was stirred and left for $1 \mathrm{~h}$ in the dark, after which the absorbance at $517 \mathrm{~nm}$ was measured using methanol as blank sample $[4,8,13]$. The DPPH radical scavenging ability of the tested compounds was calculated as the inhibition percentage (I\%) using the formula:

$$
\mathrm{I} \%=\left(\left(\mathrm{A}_{0}-\mathrm{A}_{\mathrm{t}}\right) / \mathrm{A}_{0}\right) \times 100,
$$

wherein $\mathrm{A}_{0}=$ the absorbance value of the DPPH methanolic solution of $0.1 \mathrm{mM} ; A_{t}=$ the absorbance value of the tested compounds. For each compound it was calculated the effective concentration $50\left(\mathrm{EC}_{50}\right)$ by linear regression and ascorbic acid $(1 \mathrm{mg} / \mathrm{mL})$ was used as a positive control. All determinations were performed in triplicate and the results were expressed as arithmetic average \pm standard deviation (SD). The ABTS ${ }^{++}$Radical Scavenging Assay. The ABTS ${ }^{++}$ free radical was generated by treating of the aqueous solution of 2,2'-azino-bis-(3-ethylbenzothiazoline6-sulfonic acid) (7 mM) with ammonium persulfate (2.45 $\mathrm{mM})$, after that the resulting mixture was kept in the dark for 12 to $16 \mathrm{~h}$ to promote the formation of $\mathrm{ABTS}^{\cdot+}$ free radical. The tested compounds were dissolved in DMSO to obtain a stock solution of 10 $\mathrm{mg} / \mathrm{mL}$. From the stock solution were taken different samples $(12.5 \mu \mathrm{L}, 25 \mu \mathrm{L}, 50 \mu \mathrm{L}, 100 \mu \mathrm{L}, 150 \mu \mathrm{L}$, $200 \mu \mathrm{L}$ ) which were diluted with DMSO up to the volume of $200 \mu \mathrm{L}$ and then $1800 \mu \mathrm{L}$ of $\mathrm{ABTS}^{\bullet+}$ solution was added. The concentration of the compounds tested in the obtained samples was $0.061 \mathrm{mg} / \mathrm{mL}, 0.125 \mathrm{mg} / \mathrm{mL}$, $0.25 \mathrm{mg} / \mathrm{mL}, 0.50 \mathrm{mg} / \mathrm{mL}, 0.75 \mathrm{mg} / \mathrm{mL}$ and $1.0 \mathrm{mg} / \mathrm{mL}$ respectively. The mixture was left to stand for $6 \mathrm{~min}$, after which the absorbance at $734 \mathrm{~nm}$ was measured using ethanol as blank sample [7, 14, 16, 17].

The ABTS radical scavenging ability of the tested compounds was calculated as the inhibition percentage (I\%) using the formula:

$$
\mathrm{I} \%=\left(\left(\mathrm{A}_{0}-\mathrm{A}_{\mathrm{t}}\right) / \mathrm{A}_{0}\right) \times 100,
$$

wherein $\mathrm{A}_{0}=$ the absorbance value of the ethanolic solution of $\mathrm{ABTS}^{\cdot+}, \mathrm{A}_{\mathrm{t}}=$ the absorbance value of the tested compounds. For each compound it was calculated the effective concentration $50\left(\mathrm{EC}_{50}\right)$ by linear regression and ascorbic acid $(1 \mathrm{mg} / \mathrm{mL})$ was used as a positive control. All determinations were performed in triplicate and the results were expressed as arithmetic average \pm standard deviation (SD).

\section{Results and Discussion}

Synthesis. The hydrazones derivatives of diclofenac (4a-s) were synthesized according to Figure 1. Sodium diclofenac, in the presence of $\mathrm{HCl}$, gave diclofenac, acid form (1) which was transformed into the corresponding ethyl ester (2) by reaction with ethyl alcohol. The reaction of the compound (2) with an excess of hydrazine hydrate $64 \%$ resulted diclofenac hydrazide (3). Then, the condensation of the compound 
FARMACIA, 2020, Vol. 68, 2

(3) with different aromatic aldehydes led to the formation of the corresponding hydrazones $(\mathbf{4 a}-\mathbf{s})$ in good yields. Physico-chemical and spectral characterization. The hydrazone derivatives of diclofenac (4a-s) are crystalline powders, which have a coloration that varies from white to yellow, are soluble in DMSO, sparingly soluble in acetone, insoluble in methanol, chloroform, benzen, dioxane, diethyl ether. The molecular weight calculated $(\mathrm{m} / \mathrm{z})$ and found $\left([\mathrm{M}+\mathrm{H}]^{+}\right)$ are presented in Table I together with physicochemical features (yield, melting point, Rf).

The physico-chemical and mass spectra data of hydrazone derivatives (4a-s)

\begin{tabular}{|c|c|c|c|c|c|c|}
\hline Comp. & 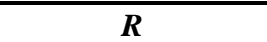 & $\eta(\%)$ & m.p. $\left({ }^{0} C\right)$ & $R f^{*}$ & $\mathrm{~m} / \mathrm{z}$ calculated & {$[\mathrm{M}+\mathrm{H}]^{+}$found } \\
\hline $4 a$ & $2-\mathrm{NO}_{2}$ & 91 & 223 & 0.39 & 442.108359 & 442.108114 \\
\hline $4 \mathrm{bb}$ & $4-\mathrm{CN}$ & 70 & 249 & 0.41 & 423.077416 & 423.077393 \\
\hline $4 c$ & $3-\mathrm{NO}_{2}$ & 82 & 234 & 0.40 & 442.108359 & 442.108217 \\
\hline 4d & $3-\mathrm{OCH}_{2} \mathrm{CH}_{3}-4-\mathrm{OH}$ & 65 & 243 & 0.37 & 458.103273 & 458.103331 \\
\hline $4 e$ & 4-Br-2-NO $\mathrm{NO}_{2}$ & 90 & 238 & 0.42 & 522.977734 & 522.977581 \\
\hline $4 f$ & $2-\mathrm{Cl}-5-\mathrm{CF}_{3}$ & 80 & 235 & 0.41 & 500.030556 & 500.030460 \\
\hline $4 g$ & $4-\mathrm{OCH}_{3}$ & 75 & 256 & 0.40 & 428.092709 & 428.092864 \\
\hline $4 \mathrm{~h}$ & $-\mathrm{H}$ & 72 & 262 & 0.40 & 398.082144 & 398.082158 \\
\hline $4 i$ & $4-\mathrm{CH}_{3}$ & 92 & 272 & 0.38 & 412.146230 & 412.146128 \\
\hline $4 j$ & $3-\mathrm{CF}_{3}$ & 82 & 261 & 0.39 & 466.069529 & 466.069573 \\
\hline $4 \mathbf{k}$ & 3,4-diF & 78 & 239 & 0.43 & 434.063300 & 434.063406 \\
\hline 41 & 3-Br-4-OH & 84 & 233 & 0.42 & 493.156794 & 493.156548 \\
\hline $4 m$ & 2,5-diBr & 71 & 287 & 0.41 & 556.156794 & 556.156508 \\
\hline $4 n$ & $2-\mathrm{Br}-4-\mathrm{F}$ & 65 & 229 & 0.43 & 495.151315 & 495.151159 \\
\hline 40 & $4-\mathrm{Br}-2-\mathrm{F}$ & 69 & 230 & 0.43 & 495.151315 & 495.151644 \\
\hline $4 p$ & $3-\mathrm{F}-4-\mathrm{CH}_{3}$ & 85 & 268 & 0.39 & 430.088372 & 430.088427 \\
\hline $4 q$ & $4-\mathrm{F}$ & 88 & 225 & 0.39 & 416.072722 & 416.072626 \\
\hline $4 r$ & $3-\mathrm{OCH}_{3}-4-\mathrm{CH}_{3}$ & 83 & 274 & 0.33 & 442.156794 & 442.156693 \\
\hline $4 s$ & 2-Br-3-OH-4-OCH & 67 & 262 & 0.41 & 523.156794 & 523.156693 \\
\hline
\end{tabular}

"petroleum ether:ethyl acetate $=8.0: 2.0 \mathrm{v} / \mathrm{v}$;

In the FT-IR spectra the appearance of the stretching band of the imine group (-N=CH-) at $1628-1474 \mathrm{~cm}^{-1}$ [6] confirms the successfully formation of the hydrazones derivatives of diclofenac. The characteristic bands of the aromatic ring appeared in the range of 3024 $2839 \mathrm{~cm}^{-1}$, and between $1580-1287 \mathrm{~cm}^{-1}$, and the phenyl ring substituents were observed at: $3194 \mathrm{~cm}^{-1}$
$(\mathrm{OH}), 2222 \mathrm{~cm}^{-1}(\mathrm{CN}), 1266-1180 \mathrm{~cm}^{-1}\left(\mathrm{OCH}_{3}\right)$, $1342-1304 \mathrm{~cm}^{-1}\left(\mathrm{NO}_{2}\right), 1297-1080 \mathrm{~cm}^{-1}(\mathrm{C}-\mathrm{F})$, 1080 - $941 \mathrm{~cm}^{-1}$ (C-Br), $887-663 \mathrm{~cm}^{-1}(\mathrm{C}-\mathrm{Cl})$.

The structure of the hydrazone derivatives is strongly supported also by the ${ }^{1} \mathrm{H}-\mathrm{NMR}$ spectra where the proton of $-\mathrm{CH}=\mathrm{N}$ - resonates as singlet, between 8.37 - $7.85 \mathrm{ppm}$ [1]. FT-IR and ${ }^{1} \mathrm{H}-\mathrm{RMN}$ data are listed in Table II.

Table II

The FT-IR and ${ }^{1} \mathrm{H}-\mathrm{RMN}$ data of hydrazone derivatives (4a-s)

\begin{tabular}{|c|c|c|}
\hline No & ${ }^{1} \mathrm{H}-\mathrm{NMR}$ signals $\delta(\mathrm{ppm})$ & FT-IR characteristic band $\left(\mathrm{cm}^{-1}\right)$ \\
\hline $4 a$ & $\begin{array}{l}3.73-4.13\left(2 \mathrm{H}, 2 \mathrm{~s}, \mathrm{CH}_{2} \mathrm{CO}\right), 6.31(1 \mathrm{H}, \mathrm{m}, \mathrm{H} 3), 6.87(1 \mathrm{H}, \mathrm{dt}, \\
\mathrm{H} 5), 7.17\left(3 \mathrm{H}, \mathrm{m}, \mathrm{H} 4, \mathrm{H}^{\prime \prime}, \mathrm{H} 5^{\prime \prime}\right), 7.64\left(4 \mathrm{H}, \mathrm{m}, \mathrm{H} 6, \mathrm{H} 3^{\prime}, \mathrm{H} 4^{\prime},\right. \\
\left.\mathrm{H}^{\prime}\right), 8.07\left(2 \mathrm{H}, \mathrm{m}, \mathrm{H} 3^{\prime \prime}, \mathrm{H}^{\prime \prime}\right), 8.37(1 \mathrm{H}, \mathrm{d}, \mathrm{CH}=\mathrm{N}), 8.65(1 \mathrm{H}, \\
\text { s, NH), } 12.01(1 \mathrm{H}, \mathrm{d}, \mathrm{CONH})\end{array}$ & $\begin{array}{l}3734,3279,3032(-\mathrm{NH}-), 2361,1580,1342 \\
\left(\mathrm{CH}_{\mathrm{Ar}}\right), 1651(-\mathrm{CO}-\mathrm{NH}), 1628(-\mathrm{CH}=\mathrm{N}), 1566 \\
1304\left(\mathrm{C}-\mathrm{NO}_{2}\right), 849,787,748,702(-\mathrm{C}-\mathrm{Cl})\end{array}$ \\
\hline $4 b$ & $\begin{array}{l}3.74-4.17\left(2 \mathrm{H}, 2 \mathrm{~s}, \mathrm{CH}_{2} \mathrm{CO}\right), 6.31(1 \mathrm{H}, \mathrm{m}, \mathrm{H} 3), 6.87(1 \mathrm{H}, \mathrm{dt}, \\
\mathrm{H} 5), 7.16\left(3 \mathrm{H}, \mathrm{m}, \mathrm{H} 4, \mathrm{H} 6, \mathrm{H} 4^{\prime}\right), 7.54\left(3 \mathrm{H}, \mathrm{m}, \mathrm{H} 3^{\prime}, \mathrm{H}^{\prime}, \mathrm{H} 5^{\prime \prime}\right), \\
7.92\left(3 \mathrm{H}, \mathrm{m}, \mathrm{H} 2^{\prime \prime}, \mathrm{H} 3^{\prime \prime}, \mathrm{H}^{\prime \prime}\right), 7.96(1 \mathrm{H}, \mathrm{s}, \mathrm{CH}=\mathrm{N}), 8.20(1 \mathrm{H}, \\
\mathrm{d}, \mathrm{NH}), 11.92(1 \mathrm{H}, \mathrm{s}, \mathrm{CONH})\end{array}$ & $\begin{array}{l}3742,3263,3040(-\mathrm{NH}-), 3001,1504,1450 \\
\left(\mathrm{CH}_{\mathrm{Ar}}\right), 2222(\mathrm{CN}), 1666(-\mathrm{CO}-\mathrm{NH}), 1589 \\
(-\mathrm{CH}=\mathrm{N}), 841,764,702(-\mathrm{C}-\mathrm{Cl})\end{array}$ \\
\hline $4 c$ & $\begin{array}{l}3.71-4.11\left(2 \mathrm{H}, 2 \mathrm{~s}, \mathrm{CH}_{2} \mathrm{CO}\right), 6.32(1 \mathrm{H}, \mathrm{m}, \mathrm{H} 3), 6.88(1 \mathrm{H}, \mathrm{dt}, \\
\mathrm{H} 5), 7.19\left(3 \mathrm{H}, \mathrm{m}, \mathrm{H} 4, \mathrm{H}^{\prime \prime}, \mathrm{H} 5^{\prime \prime}\right), 7.64\left(4 \mathrm{H}, \mathrm{m}, \mathrm{H} 6, \mathrm{H} 3^{\prime}, \mathrm{H} 4^{\prime},\right. \\
\left.\mathrm{H}^{\prime}\right), 8.07\left(2 \mathrm{H}, \mathrm{m}, \mathrm{H} 2^{\prime \prime}, \mathrm{H} 6^{\prime \prime}\right), 8.37(1 \mathrm{H}, \mathrm{d}, \mathrm{CH}=\mathrm{N}), 8.65(1 \mathrm{H}, \\
\mathrm{s}, \mathrm{NH}), 12.09(1 \mathrm{H}, \mathrm{d}, \mathrm{CONH})\end{array}$ & $\begin{array}{l}3734,3271,3024(-\mathrm{NH}-), 2361,1583,1515 \\
\left(\mathrm{CH}_{\mathrm{Ar}}\right), 1651(-\mathrm{CO}-\mathrm{NH}), 1528(-\mathrm{CH}=\mathrm{N}), 1566 \\
1304\left(\mathrm{C}-\mathrm{NO}_{2}\right), 872,810,741,679(-\mathrm{C}-\mathrm{Cl})\end{array}$ \\
\hline
\end{tabular}


FARMACIA, 2020, Vol. 68, 2

\begin{tabular}{|c|c|c|}
\hline No & ${ }^{1} \mathrm{H}-\mathrm{NMR}$ signals $\delta(\mathrm{ppm})$ & FT-IR characteristic band (c \\
\hline $4 d$ & $\begin{array}{l}1.35\left(3 \mathrm{H}, \mathrm{m}, \mathrm{CH}_{3}\right), 3.68-4.11\left(2 \mathrm{H}, 2 \mathrm{~s}, \mathrm{CH}_{2} \mathrm{CO}\right), 4.03(2 \mathrm{H}, \mathrm{dq} \\
\left.\mathrm{OCH}_{2}\right), 6.30(1 \mathrm{H}, \mathrm{dd}, \mathrm{H} 3), 6.85\left(2 \mathrm{H}, \mathrm{m}, \mathrm{H} 5, \mathrm{H} 5^{\prime \prime}\right), 7.17(5 \mathrm{H}, \mathrm{m}, \\
\left.\mathrm{H} 6, \mathrm{H} 4, \mathrm{H} 4^{\prime}, \mathrm{H} 2^{\prime \prime}, \mathrm{H} 6^{\prime \prime}\right), 7.53\left(2 \mathrm{H}, \mathrm{m}, \mathrm{H} 2^{\prime}, \mathrm{H} 5^{\prime}\right), 7.83(1 \mathrm{H}, \mathrm{d}, \\
\mathrm{CH}=\mathrm{N}), 8.12(1 \mathrm{H}, \mathrm{d}, \mathrm{NH}), 9.45(1 \mathrm{H}, \mathrm{d}, \mathrm{OH}), 11.54(1 \mathrm{H}, \mathrm{d}, \mathrm{CONH})\end{array}$ & $\begin{array}{l}3734,3518,3286(-\mathrm{NH}-), 3194(-\mathrm{OH}), 2361, \\
1512,1443\left(\mathrm{CH}_{\mathrm{Ar}}\right), 1643(-\mathrm{CO}-\mathrm{NH}-), 1512 \\
(-\mathrm{CH}=\mathrm{N}), 1180(\mathrm{O}-\mathrm{C}), 864,833,748,717 \\
(-\mathrm{C}-\mathrm{Cl})\end{array}$ \\
\hline $4 e$ & $\begin{array}{l}3.69 \text { - } 4.08\left(2 \mathrm{H}, 2 \mathrm{~s}, \mathrm{CH}_{2} \mathrm{CO}\right), 6.27(1 \mathrm{H}, \mathrm{m}, \mathrm{H} 3), 6.83(1 \mathrm{H}, \mathrm{m}, \\
\mathrm{H} 5), 7.14\left(3 \mathrm{H}, \mathrm{m}, \mathrm{H} 4, \mathrm{H} 6, \mathrm{H} 4{ }^{\prime}\right), 7.49\left(3 \mathrm{H}, \mathrm{m}, \mathrm{H} 2^{\prime}, \mathrm{H}^{\prime}, \mathrm{H} 6^{\prime \prime}\right), \\
7.96\left(2 \mathrm{H}, \mathrm{m}, \mathrm{H} 3^{\prime \prime}, \mathrm{H} 5^{\prime \prime}\right), 8.25(1 \mathrm{H}, \mathrm{m}, \mathrm{CH}=\mathrm{N}), 8.43(1 \mathrm{H}, \mathrm{d}, \\
\mathrm{NH}), 12.01(1 \mathrm{H}, \mathrm{d}, \mathrm{CONH})\end{array}$ & $\begin{array}{l}3734,3271,3186(-\mathrm{NH}-), 2993,1529,1257 \\
\left(\mathrm{CH}_{\mathrm{Ar}}\right), 1649(-\mathrm{CO}-\mathrm{NH}), 1574(-\mathrm{CH}=\mathrm{N}) \\
1450,1342\left(\mathrm{C}-\mathrm{NO}_{2}\right), 995(-\mathrm{C}-\mathrm{Br}), 879,748 \\
687(-\mathrm{C}-\mathrm{Cl})\end{array}$ \\
\hline $4 f$ & $\begin{array}{l}3.70 \text { - } 4.12\left(2 \mathrm{H}, 2 \mathrm{~s}, \mathrm{CH}_{2} \mathrm{CO}\right), 6.26(1 \mathrm{H}, \mathrm{dd}, \mathrm{H} 3), 6.82(1 \mathrm{H}, \mathrm{m}, \\
\mathrm{H} 5), 7.12\left(3 \mathrm{H}, \mathrm{t}, \mathrm{H} 4, \mathrm{H} 3^{\prime \prime}, \mathrm{H} 4^{\prime \prime}\right), 7.45\left(2 \mathrm{H}, \mathrm{m}, \mathrm{H} 6, \mathrm{H} 4^{\prime}\right), 7.77 \\
\left(3 \mathrm{H}, \mathrm{m}, \mathrm{H} 3^{\prime}, \mathrm{H} 5^{\prime}, \mathrm{H} 6^{\prime \prime}\right), 8.12(1 \mathrm{H}, \mathrm{d}, \mathrm{CH}=\mathrm{N}), 8.51(1 \mathrm{H}, 2 \mathrm{~s}, \mathrm{NH}), \\
12.03(1 \mathrm{H}, 2 \mathrm{~s}, \mathrm{CONH})\end{array}$ & $\begin{array}{l}3302,3178(-\mathrm{NH}-), 3024,1566,1504\left(\mathrm{CH}_{\mathrm{Ar}}\right), \\
1659 \text { (-CO-NH-), } 1504(-\mathrm{CH}=\mathrm{N}), 1257,1149, \\
1080 \text { (C-F), 825, 748, 717, } 663(-\mathrm{C}-\mathrm{Cl})\end{array}$ \\
\hline $4 g$ & $\begin{array}{l}2.04\left(3 \mathrm{H}, \mathrm{s}, \mathrm{CH}_{3}\right), 3.65-4.07\left(2 \mathrm{H}, 2 \mathrm{~s}, \mathrm{CH}_{2} \mathrm{CO}\right), 6.26(1 \mathrm{H}, \mathrm{m}, \\
\mathrm{H} 3), 6.82(1 \mathrm{H}, \mathrm{m}, \mathrm{H} 5), 7.08\left(5 \mathrm{H}, \mathrm{m}, \mathrm{H} 4, \mathrm{H} 6, \mathrm{H} 4^{\prime}, \mathrm{H} 2^{\prime \prime}, \mathrm{H}^{\prime \prime}\right), \\
7.55\left(4 \mathrm{H}, \mathrm{m}, \mathrm{H} 3^{\prime}, \mathrm{H} 5^{\prime}, \mathrm{H} 3^{\prime \prime}, \mathrm{H} 5^{\prime \prime}\right), 7.85(1 \mathrm{H}, \mathrm{d}, \mathrm{CH}=\mathrm{N}), 8.12 \\
(1 \mathrm{H}, \mathrm{d}, \mathrm{NH}), 11.56(1 \mathrm{H}, \mathrm{d}, \mathrm{CONH})\end{array}$ & $\begin{array}{l}3256,3009(-\mathrm{NH}-), 2901,1512,1450\left(\mathrm{CH}_{\mathrm{Ar}}\right) \text {, } \\
1643(-\mathrm{CO}-\mathrm{NH}-), 1597(-\mathrm{CH}=\mathrm{N}), 1257(\mathrm{O}-\mathrm{C}) \text {, } \\
833,756,717,679(-\mathrm{C}-\mathrm{Cl})\end{array}$ \\
\hline $4 h$ & $\begin{array}{l}3.71 \text { - } 4.14\left(2 \mathrm{H}, 2 \mathrm{~s}, \mathrm{CH}_{2} \mathrm{CO}\right), 6.31(1 \mathrm{H}, \mathrm{dd}, \mathrm{H} 3), 6.87(1 \mathrm{H}, \mathrm{dt} \\
\mathrm{H} 5), 7.19\left(3 \mathrm{H}, \mathrm{m}, \mathrm{H} 4, \mathrm{H} 6, \mathrm{H} 4{ }^{\prime}\right), 7.50\left(5 \mathrm{H}, \mathrm{m}, \mathrm{H} 3^{\prime}, \mathrm{H} 5^{\prime}, \mathrm{H} 3^{\prime \prime}\right. \\
\left.\mathrm{H} 4^{\prime \prime}, \mathrm{H} 5^{\prime \prime}\right), 7.74\left(2 \mathrm{H}, \mathrm{dd}, \mathrm{H} 2^{\prime \prime}, \mathrm{H} 6^{\prime \prime}\right), 8.07(1 \mathrm{H}, \mathrm{d}, \mathrm{CH}=\mathrm{N}), 8.24 \\
(1 \mathrm{H}, \mathrm{s}, \mathrm{NH}), 11.73(1 \mathrm{H}, \mathrm{d}, \mathrm{CONH})\end{array}$ & $\begin{array}{l}3286,3016(-\mathrm{NH}-), 1504,1450\left(\mathrm{CH}_{\mathrm{Ar}}\right), 1643 \\
(-\mathrm{CO}-\mathrm{NH}), 1574(-\mathrm{CH}=\mathrm{N}), 748,694,687 \\
(-\mathrm{C}-\mathrm{Cl})\end{array}$ \\
\hline $4 i$ & $\begin{array}{l}2.41\left(3 \mathrm{H}, \mathrm{s}, \mathrm{CH}_{3}\right), 3.70-4 \\
\mathrm{H} 3), 6.86(1 \mathrm{H}, \mathrm{dt}, \mathrm{H} 5), 7 . \\
7.58\left(4 \mathrm{H}, \mathrm{m}, \mathrm{H} 3^{\prime}, \mathrm{H}^{\prime}, \mathrm{H} 3\right. \\
(1 \mathrm{H}, \mathrm{d}, \mathrm{NH}), 11.66(1 \mathrm{H}, \mathrm{c}\end{array}$ & $\begin{array}{l}3256,3178(-\mathrm{NH}-), 2901,1504,1450\left(\mathrm{CH}_{\mathrm{Ar}}\right) \\
1643(-\mathrm{CO}-\mathrm{NH}-), 1566(-\mathrm{CH}=\mathrm{N}), 818,748, \\
710,679(-\mathrm{C}-\mathrm{Cl})\end{array}$ \\
\hline $4 \mathbf{j}$ & $\begin{array}{l}(1 \mathrm{H}, \mathrm{dd}, \mathrm{H} 3), 6.87(1 \mathrm{H}, \mathrm{dt} \\
62\left(5 \mathrm{H}, \mathrm{m}, \mathrm{H} 3^{\prime}, \mathrm{H}^{\prime}, \mathrm{H}^{\prime \prime}\right. \\
11(1 \mathrm{H}, \mathrm{d}, \mathrm{CH}=\mathrm{N}), 8.33 \\
\end{array}$ & $\begin{array}{l}3279,3194(-\mathrm{NH}- \\
1651 \text { (-CO-NH-), } \\
1157 \text { (C-F), 771, }\end{array}$ \\
\hline $4 k$ & $\begin{array}{l}\mathrm{H}, \mathrm{dd}, \mathrm{H} 3), 6.87(1 \mathrm{H}, \mathrm{dt}, \mathrm{H} 5) \\
\mathrm{H}, \mathrm{m}, \mathrm{H} 2^{\prime}, \mathrm{H} 5^{\prime}, \mathrm{H} 2^{\prime \prime}, \mathrm{H} 5^{\prime \prime} \\
\mathrm{l}, \mathrm{NH}), 11.83(1 \mathrm{H}, \mathrm{d}, \mathrm{CONH})\end{array}$ & $\begin{array}{l}\left(\mathrm{CH}_{\mathrm{Ar}}\right) \\
1,1203 \\
1)\end{array}$ \\
\hline 41 & $\begin{array}{l}\text { 6.30 (1H, dd, H3), 6.86 (1H, dt, } \\
\left.4^{\prime}, \mathrm{H} 2^{\prime \prime}\right), 7.62\left(4 \mathrm{H}, \mathrm{m}, \mathrm{H} 3^{\prime}, \mathrm{H} 5^{\prime}\right. \\
\mathrm{N}), 8.11(1 \mathrm{H}, \mathrm{d}, \mathrm{NH}), 10.76(1 \mathrm{H} \\
\text { I) }\end{array}$ & $\begin{array}{l}3495,3279(-\mathrm{NH}-), 3186(-\mathrm{OH}), 3001,1497, \\
1443\left(\mathrm{CH}_{\mathrm{Ar}}\right), 1643(-\mathrm{CO}-\mathrm{NH}-), 1589(-\mathrm{CH}=\mathrm{N}), \\
1080(-\mathrm{C}-\mathrm{Br}), 818,748,710,671(-\mathrm{C}-\mathrm{Cl})\end{array}$ \\
\hline $4 m$ & $\begin{array}{l}3.70 \text { - } 4.12\left(2 \mathrm{H}, 2 \mathrm{~s}, \mathrm{CH}_{2} \mathrm{CO}\right), 6.26(1 \mathrm{H}, \mathrm{dd}, \mathrm{H} 3), 6.82(1 \mathrm{H}, \mathrm{m}, \\
\mathrm{H} 5), 7.12\left(3 \mathrm{H}, \mathrm{t}, \mathrm{H} 4, \mathrm{H} 3^{\prime \prime}, \mathrm{H}^{\prime \prime}\right), 7.45\left(2 \mathrm{H}, \mathrm{m}, \mathrm{H} 6, \mathrm{H} 4^{\prime}\right), 7.77 \\
\left(3 \mathrm{H}, \mathrm{m}, \mathrm{H} 3^{\prime}, \mathrm{H} 5^{\prime}, \mathrm{H} 6^{\prime \prime}\right), 8.12(1 \mathrm{H}, \mathrm{d}, \mathrm{CH}=\mathrm{N}), 8.51(1 \mathrm{H}, 2 \mathrm{~s}, \mathrm{NH}) \\
12.03(1 \mathrm{H}, 2 \mathrm{~s}, \mathrm{CONH})\end{array}$ & $\begin{array}{l}1497,1443\left(\mathrm{CH}_{\mathrm{Ar}}\right) \\
\mathrm{CH}=\mathrm{N}), 1080,1026 \\
\mathrm{C}-\mathrm{Cl})\end{array}$ \\
\hline $4 n$ & $\begin{array}{l}3.69 \text { - } 4.08\left(2 \mathrm{H}, 2 \mathrm{~s}, \mathrm{CH}_{2} \mathrm{CO}\right), 6.27(1 \mathrm{H}, \mathrm{m}, \mathrm{H} 3), 6.83(1 \mathrm{H}, \mathrm{m}, \\
\mathrm{H} 5), 7.14\left(3 \mathrm{H}, \mathrm{m}, \mathrm{H} 4, \mathrm{H} 6, \mathrm{H} 4{ }^{\prime}\right), 7.49\left(3 \mathrm{H}, \mathrm{m}, \mathrm{H} 2^{\prime}, \mathrm{H}^{\prime}, \mathrm{H} 6^{\prime \prime}\right) \\
7.96\left(2 \mathrm{H}, \mathrm{m}, \mathrm{H} 3^{\prime \prime}, \mathrm{H} 5^{\prime \prime}\right), 8.25(1 \mathrm{H}, \mathrm{m}, \mathrm{CH}=\mathrm{N}), 8.43(1 \mathrm{H}, \mathrm{d}, \\
\mathrm{NH}), 12.01(1 \mathrm{H}, \mathrm{d}, \mathrm{CONH})\end{array}$ & $\begin{array}{l}3205,3101(-\mathrm{NH}-), 2361,1389\left(\mathrm{CH}_{\mathrm{Ar}}\right), 1589 \\
(-\mathrm{CO}-\mathrm{NH}), 1474(-\mathrm{CH}=\mathrm{N}), 1281,1203,1180 \\
(\mathrm{C}-\mathrm{F}), 1034,941(-\mathrm{C}-\mathrm{Br}), 887,856,818,663 \\
(-\mathrm{C}-\mathrm{Cl})\end{array}$ \\
\hline 40 & $\begin{array}{l}3.70 \text { - } 4.08\left(2 \mathrm{H}, 2 \mathrm{~s}, \mathrm{CH}_{2} \mathrm{CO}\right), 6.28(1 \mathrm{H}, \mathrm{m}, \mathrm{H} 3), 6.83(1 \mathrm{H}, \mathrm{m}, \\
\mathrm{H} 5), 7.14\left(3 \mathrm{H}, \mathrm{m}, \mathrm{H} 4, \mathrm{H} 6, \mathrm{H} 4{ }^{\prime}\right), 7.50\left(3 \mathrm{H}, \mathrm{m}, \mathrm{H} 2^{\prime}, \mathrm{H}^{\prime}, \mathrm{H} 6^{\prime \prime}\right) \\
7.96\left(2 \mathrm{H}, \mathrm{m}, \mathrm{H} 3^{\prime \prime}, \mathrm{H} 5^{\prime \prime}\right), 8.25(1 \mathrm{H}, \mathrm{m}, \mathrm{CH}=\mathrm{N}), 8.43(1 \mathrm{H}, \mathrm{d}, \\
\mathrm{NH}), 12.02(1 \mathrm{H}, \mathrm{d}, \mathrm{CONH})\end{array}$ & $\begin{array}{l}3308,3070(-\mathrm{NH}-), 1404,1389\left(\mathrm{CH}_{\mathrm{Ar}}\right), 1620 \\
(-\mathrm{CO}-\mathrm{NH}), 1597(-\mathrm{CH}=\mathrm{N}), 1265,1211(\mathrm{C}-\mathrm{F}) \text {, } \\
1057,957(-\mathrm{C}-\mathrm{Br}), 879,839,671(-\mathrm{C}-\mathrm{Cl})\end{array}$ \\
\hline $4 p$ & $\begin{array}{l}2.27\left(3 \mathrm{H}, \mathrm{s}, \mathrm{CH}_{3}\right), 3.71-4.14\left(2 \mathrm{H}, 2 \mathrm{~s}, \mathrm{CH}_{2} \mathrm{CO}\right), 6.31(1 \mathrm{H}, \mathrm{dd} \\
\mathrm{H} 3), 6.87(1 \mathrm{H}, \mathrm{m}, \mathrm{H} 5), 7.22\left(4 \mathrm{H}, \mathrm{m}, \mathrm{H} 4, \mathrm{H} 2^{\prime \prime}, \mathrm{H} 5^{\prime \prime}, \mathrm{H}^{\prime \prime}\right), 7.53 \\
\left(4 \mathrm{H}, \mathrm{m}, \mathrm{H} 6, \mathrm{H} 3^{\prime}, \mathrm{H} 4^{\prime}, \mathrm{H}^{\prime}\right), 8.03(1 \mathrm{H}, \mathrm{d}, \mathrm{CH}=\mathrm{N}), 8.20(1 \mathrm{H}, 2 \mathrm{~s}, \\
\mathrm{NH}), 11.76(1 \mathrm{H}, \mathrm{d}, \mathrm{CONH})\end{array}$ & $\begin{array}{l}3263,3171,3001(-\mathrm{NH}-), 2901,1504,1450 \\
\left(\mathrm{CH}_{\mathrm{Ar}}\right), 1643(-\mathrm{CO}-\mathrm{NH}), 1566(\mathrm{CH}=\mathrm{N}), 1265, \\
1188,1157(\mathrm{C}-\mathrm{F}), 872,833,771,656(-\mathrm{C}-\mathrm{Cl})\end{array}$ \\
\hline $4 q$ & $\begin{array}{l}3.70 \text { - } 4.13\left(2 \mathrm{H}, 2 \mathrm{~s}, \mathrm{CH}_{2} \mathrm{CO}\right), 6.31(1 \mathrm{H}, \mathrm{dd}, \mathrm{H} 3), 6.86(1 \mathrm{H}, \mathrm{dt}, \\
\mathrm{H} 5), 7.06(1 \mathrm{H}, \mathrm{q}, \mathrm{H} 4), 7.25\left(4 \mathrm{H}, \mathrm{m}, \mathrm{H} 6, \mathrm{H}^{\prime}, \mathrm{H} 3^{\prime \prime}, \mathrm{H} 5^{\prime \prime}\right), 7.53 \\
\left(2 \mathrm{H}, \mathrm{m}, \mathrm{H} 3^{\prime}, \mathrm{H} 5^{\prime}\right), 7.79\left(2 \mathrm{H}, \mathrm{m}, \mathrm{H} 2^{\prime \prime}, \mathrm{H} 6^{\prime \prime}\right), 8.06(1 \mathrm{H}, \mathrm{d}, \mathrm{CH}=\mathrm{N}), \\
8.24(1 \mathrm{H}, \mathrm{s}, \mathrm{NH}), 11.73(1 \mathrm{H}, \mathrm{d}, \mathrm{CONH})\end{array}$ & $\begin{array}{l}3340,3070(-\mathrm{NH}-), 2962,1582\left(\mathrm{CH}_{\mathrm{Ar}}\right), 1660 \\
(-\mathrm{CO}-\mathrm{NH}-), 1504(-\mathrm{CH}=\mathrm{N}), 1296,1203,1165 \\
(\mathrm{C}-\mathrm{F}), 856,810,764,656(-\mathrm{C}-\mathrm{Cl})\end{array}$ \\
\hline
\end{tabular}


FARMACIA, 2020, Vol. 68, 2

\begin{tabular}{|c|c|c|}
\hline No & ${ }^{1} \mathrm{H}-\mathrm{NMR}$ signals $\delta$ (ppm) & FT-IR characteristic band $\left(\mathrm{cm}^{-1}\right)$ \\
\hline $4 r$ & $\begin{array}{l}2.18\left(3 \mathrm{H}, \mathrm{s}, \mathrm{CH}_{3}\right), 3.71-4.14\left(2 \mathrm{H}, 2 \mathrm{~s}, \mathrm{CH}_{2} \mathrm{CO}\right), 3.83(3 \mathrm{H}, \mathrm{d}, \\
\left.\mathrm{OCH}_{3}\right), 6.31(1 \mathrm{H}, \mathrm{dd}, \mathrm{H} 3), 6.87(1 \mathrm{H}, \mathrm{dt}, \mathrm{H} 5), 7.21(6 \mathrm{H}, \mathrm{m}, \mathrm{H} 4, \\
\left.\mathrm{H} 6, \mathrm{H} 4^{\prime}, \mathrm{H} 2^{\prime \prime}, \mathrm{H} 5^{\prime \prime}, \mathrm{H} 6^{\prime \prime}\right), 7.56\left(2 \mathrm{H}, \mathrm{m}, \mathrm{H} 3^{\prime}, \mathrm{H} 5^{\prime}\right), 8.04(1 \mathrm{H}, \mathrm{d}, \\
\mathrm{CH}=\mathrm{N}), 8.20(1 \mathrm{H}, 2 \mathrm{~s}, \mathrm{NH}), 11.69(1 \mathrm{H}, \mathrm{d}, \mathrm{CONH})\end{array}$ & $\begin{array}{l}3286,3001(-\mathrm{NH}-), 2908,1574,1450\left(\mathrm{CH}_{\mathrm{Ar}}\right), \\
1643(-\mathrm{CO}-\mathrm{NH}-), 1504(-\mathrm{CH}=\mathrm{N}), 1266(\mathrm{O}-\mathrm{C}) \text {, } \\
810,748,710,663(-\mathrm{C}-\mathrm{Cl})\end{array}$ \\
\hline $4 s$ & $\begin{array}{l}3.73-4.14\left(2 \mathrm{H}, 2 \mathrm{~s}, \mathrm{CH}_{2} \mathrm{CO}\right), 3.85\left(3 \mathrm{H}, \mathrm{d}, \mathrm{OCH}_{3}\right), 6.34(1 \mathrm{H}, \\
\mathrm{dd}, \mathrm{H} 3), 6.89(1 \mathrm{H}, \mathrm{dt}, \mathrm{H} 5), 7.23\left(5 \mathrm{H}, \mathrm{m}, \mathrm{H} 4, \mathrm{H} 6, \mathrm{H}^{\prime}, \mathrm{H} 5^{\prime \prime},\right. \\
\left.\mathrm{H}^{\prime \prime}\right), 7.59\left(2 \mathrm{H}, \mathrm{m}, \mathrm{H} 3^{\prime}, \mathrm{H} 5^{\prime}\right), 8.06(1 \mathrm{H}, \mathrm{d}, \mathrm{CH}=\mathrm{N}), 8.22(1 \mathrm{H}, \\
2 \mathrm{~s}, \mathrm{NH}), 10.78(1 \mathrm{H}, \mathrm{d}, \mathrm{OH}), 11.72(1 \mathrm{H}, \mathrm{d}, \mathrm{CONH})\end{array}$ & $\begin{array}{l}3263,3032(-\mathrm{NH}-), 3117(-\mathrm{OH}), 2839,2361, \\
1279\left(\mathrm{CH}_{\mathrm{Ar}}\right), 1597(-\mathrm{CO}-\mathrm{NH}-), 1489(-\mathrm{CH}=\mathrm{N}) \\
1211(\mathrm{O}-\mathrm{C}), 1026(-\mathrm{C}-\mathrm{Br}), 825,663(-\mathrm{C}-\mathrm{Cl})\end{array}$ \\
\hline
\end{tabular}

The antioxidant effects evaluation

The DPPH Radical Scavenging Ability. From the obtained results (Table III) it could be observed that all tested compounds showed improved DPPH radical scavenging ability in reference with diclofenac, for which the $\mathrm{EC}_{50}$ was $1.71 \pm 0.023$. The most active compounds are $\mathbf{4 d}\left(\mathrm{EC}_{50}=0.06 \pm 0.004\right)$ and $\mathbf{4 s}$ $\left(\mathrm{EC}_{50}=0.07 \pm 0.005\right)$ which were obtained by condensation of diclofenac hydrazide (3) with 3- ethoxy-4-hydroxybenzaldehyde (4d) and 2-bromo-3hydroxy-4-methoxybenzaldehyde (4s) respectively. Appreciable anti-radicalic activity showed also $\mathbf{4 c}$ $\left(\mathrm{EC}_{50}=0.23 \pm 0.012\right), \mathbf{4 n}\left(\mathrm{EC}_{50}=0.28 \pm 0.002\right), \mathbf{4 h}$ $\left(\mathrm{EC}_{50}=0.35 \pm 0.015\right), \mathbf{4 r}\left(\mathrm{EC}_{50}=0.36 \pm 0.008\right), \mathbf{4 l}$ $\left(\mathrm{EC}_{50}=0.37 \pm 0.013\right)$ and $\mathbf{4 j}\left(\mathrm{EC}_{50}=0.40 \pm 0.014\right)$. Compared to ascorbic acid, used as a positive control, all tested compounds were less active in similar conditions.

Table III

The DPPH radical scavenging ability $\left(\mathrm{EC}_{50}, \mathrm{mg} / \mathrm{mL}\right)$ of hydrazone derivatives (4a-s)

\begin{tabular}{|c|c|c|c|c|c|}
\hline No & $E C_{50} \mathrm{mg} / \mathrm{mL}$ & No & $E C_{50} \mathrm{mg} / \mathrm{mL}$ & No & $E C_{50} \mathrm{mg} / \mathrm{mL}$ \\
\hline Diclofenac & $1.71 \pm 0.023$ & $4 \mathrm{~g}$ & $1.42 \pm 0.033$ & $4 n$ & $0.28 \pm 0.002$ \\
\hline $4 a$ & $2.33 \pm 0.018$ & $4 h$ & $0.35 \pm 0.015$ & 40 & $1.56 \pm 0.038$ \\
\hline $4 b$ & $2.02 \pm 0.045$ & $4 i$ & $0.96 \pm 0.041$ & $4 p$ & $1.31 \pm 0.031$ \\
\hline $4 c$ & $0.23 \pm 0.012$ & $4 j$ & $0.40 \pm 0.014$ & $4 q$ & $2.14 \pm 0.026$ \\
\hline 4d & $0.06 \pm 0.004$ & $4 k$ & $2.23 \pm 0.022$ & $4 r$ & $0.36 \pm 0.008$ \\
\hline $4 e$ & $1.65 \pm 0.012$ & 41 & $0.37 \pm 0.013$ & $4 s$ & $0.07 \pm 0.005$ \\
\hline $4 f$ & $2.65 \pm 0.042$ & $4 m$ & $2.33 \pm 0.026$ & $\mathbf{A A}$ & $0.006 \pm 0,004$ \\
\hline
\end{tabular}

AA - ascorbic acid; Data are mean \pm SD $(n=3, p<0.05)$

The ABTS $^{\bullet+}$ Radical Scavenging Ability. From the results obtained (Table IV) it could be observed that the most part of tested compounds showed improved $\mathrm{ABTS}^{\circ+}$ radical scavenging ability in reference with diclofenac, for which the $\mathrm{EC}_{50}$ was $0.62 \pm 0.027$. The most active compounds are $\mathbf{4 d}\left(\mathrm{EC}_{50}=0.07 \pm 0.004\right)$ and $4 \mathbf{s}\left(\mathrm{EC}_{50}=0.08 \pm 0.003\right)$ which were obtained by condensation of diclofenac hydrazide (3) with 3ethoxy-4-hydroxybenzaldehyde (4d) and 2-bromo- 3-hydroxy-4-methoxybenzaldehyde (4s) respectively. Appreciable anti-radicalic activity showed also $\mathbf{4 p}$ $\left(\mathrm{EC}_{50}=0.12 \pm 0.001\right), \mathbf{4 g}\left(\mathrm{EC}_{50}=0.12 \pm 0.007\right), \mathbf{4 l}$ $\left(\mathrm{EC}_{50}=0.13 \pm 0.008\right), \mathbf{4 r}\left(\mathrm{EC}_{50}=0.19 \pm 0.008\right), \mathbf{4 h}$ $\left(\mathrm{EC}_{50}=0.21 \pm 0.010\right)$ and $4 \mathbf{c}\left(\mathrm{EC}_{50}=0.31 \pm 0.015\right)$. Compared to ascorbic acid, used as a positive control, all tested compounds were less active in similar conditions.

Table IV

The ABTS radical scavenging ability $\left(\mathrm{EC}_{50}, \mathrm{mg} / \mathrm{mL}\right)$ of hydrazone derivatives $(\mathbf{4 a}-\mathbf{s})$

\begin{tabular}{|c|c|c|c|c|c|}
\hline No & $E C_{50} \mathrm{mg} / \mathrm{mL}$ & No & $E C_{50} \mathrm{mg} / \mathrm{mL}$ & No & $E C_{50} \mathrm{mg} / \mathrm{mL}$ \\
\hline Diclofenac & $0.62 \pm 0.027$ & $4 g$ & $0.12 \pm 0.007$ & $4 n$ & $1.03 \pm 0.022$ \\
\hline $4 a$ & $0.67 \pm 0.020$ & $4 h$ & $0.21 \pm 0.010$ & 40 & $1.06 \pm 0.038$ \\
\hline $4 \mathrm{~b}$ & $0.48 \pm 0.017$ & $4 i$ & $0.38 \pm 0.014$ & $4 p$ & $0.12 \pm 0.001$ \\
\hline $4 c$ & $0.31 \pm 0.015$ & $4 \mathbf{j}$ & $0.30 \pm 0.020$ & $4 q$ & $1.08 \pm 0.026$ \\
\hline 4d & $0.07 \pm 0.004$ & $4 k$ & $0.39 \pm 0.009$ & $4 r$ & $0.19 \pm 0.008$ \\
\hline $4 e$ & $1.72 \pm 0.048$ & 41 & $0.13 \pm 0.008$ & $4 s$ & $0.08 \pm 0.003$ \\
\hline $4 f$ & $0.74 \pm 0.018$ & $4 \mathrm{~m}$ & $0.98 \pm 0.029$ & $\mathbf{A A}$ & $0.005 \pm 0,004$ \\
\hline
\end{tabular}

AA - ascorbic acid; Data are mean \pm SD $(n=3, p<0.05)$

\section{Conclusions}

A series of 19 hydrazones of diclofenac (4a-s) has been synthesized and optimal conditions of reaction were established. All the synthetized compounds were characterized in terms of solubility in different organic solvents, melting point, yield, molecular formula, and the chemical structure was proved using FT-IR, ${ }^{1} \mathrm{H}-\mathrm{NMR}$ and HR-MS spectroscopy. The evaluation of the antioxidant potential of hydrazone derivatives was performed using two in vitro methods: DPPH and ABTS radical scavenging assay. The results obtained support that the antioxidant effect of tested compounds 
increases with the concentration and is influenced by the nature of the substituent on the aromatic ring. The most active compounds were $\mathbf{4 d}$ and $\mathbf{4 s}$, which were obtained by condensation of diclofenac hydrazide with 3-ethoxy-4-hydroxy-benzaldehyde and 2-bromo3-hydroxy-4-methoxybenzaldehyde respectively, which showed the lowest values of $\mathrm{EC}_{50}$, for both free radical assays. The results showed that the chemical modulation of diclofenac by introducing a imine group has a favourable influence on antioxidant potential of the synthetized compounds.

\section{Acknowledgement}

Scientific research was funded by "Grigore T. Popa" University of Medicine and Pharmacy Iași, Romania, based on contract no. 30341/28.12.2017 and by the grant of UEFISCDI, PN III Program, AUF-RO, AUF-IFA 2019-2020, contract no. 28/2019.

\section{Conflict of interest}

The authors declare no conflict of interest.

\section{References}

1. Abd El Wahed MG, Bayoumi HA, Mohammed MI, Physical properties of some acetylbenzaldehydehydrazone metal complexes. BKCS, 2003; 24(9): 1313-1318.

2. Al-Turki DA, Abou-Zeid, Shehata IA, Al-Omar MA, Therapeutic and toxic effects of new NSAIDs and related compounds: A review and prospective study. Int J Pharmacol., 2010; 6(6): 813-825.

3. Alam MS, Choi JH, Lee DU, Synthesis of novel Schiff base analogues of 4-amino-1,5-dimethyl-2phenylpyrazol-3-one and their evaluation for antioxidant and anti-inflammatory activity. Bioorg Med Chem., 2012; 20(13): 4103-4108.

4. Boligon AA, Machado MM, Athayde ML, Technical evaluation of antioxidant activity. Med Chem., 2014; 4(7): 517-522.

5. Bruno A, Tacconelli S, Patrignani P, Variability in reponse to non-steroidal antiinflammatory drugs: Mechanism and perspectives. Basic Clin Pharmacol Toxicol., 2014; 114: 56-63.

6. Constantin S, Panzariu A, Vasincu I, Apotrosoaei M, Confederat L, Buron F, Routier S, Profire L, Synthesis and evaluation of antioxidant activity of some hydrazones with xanthine structure. Rev Med Chir Soc Med Nat., 2015; 119(3): 910-916.
7. Floegel A, Dae-Ok K, Sang-Jin C, Sung IK, Chun OK, Comparison of ABTS/DPPH assays to measure antioxidant capacity in popular antioxidant-rich US foods. J Food Compos Anal., 2011; 24: 1043-1048.

8. Iacob A, Drăgan M, Constantin S, Lupaşcu F, Confederat L, Buron F, Routier S, Profire L, Evaluation of antioxidant activity of some imines derivatives of L-arginine. Rev Med Chir Soc Med Nat., 2016; 120(2): 439-444.

9. Pânzariu A, Vasincu IM, Dragostin OM, Drăgan M, Buron F, Routier F, Profire L, New arginine derivatives synthesis and biological evaluation. Farmacia, 2015; 63(4): 581-585.

10. Pountos I, Georgouli T, Bird H, Giannoudis PV, Nonsteroidal anti-inflammatory drugs: prostaglandins, indications, and side effects. Int J Interferon Cytokine Mediat Res., 2011; 2011(3): 19-27.

11. Rao PPN, Kabir SN, Mohamed T, Nonsteroidal AntiInflammatory Drugs (NSAIDs): Progress in Small Molecule Drug Development. Pharmaceuticals, 2010; 3(5): 1530-1549.

12. Rao PPN, Knaus EE, Evolution of Nonsteroidal AntiInflammatory Drugs (NSAID): cyclooxygenase (COX) inhibition and beyond. J Pharm Pharmaceut Sci., 2008; 11(2): 81-110.

13. Rashid MA, Akhtar MN, Ashraf A, Nazir S, Ijaz A, Omar NA, Noor S, Basit A, Tareen RB, Chemical composition and antioxidant, antimicrobial and haemolytic activities of Crambe cordifolia roots. Farmacia, 2018; 66(1): 165-171.

14. Re R, Pellegrini N, Protrggente A, Pannala A, Yang $\mathrm{M}$, Rice-Evans C, Antioxidant activity applying an improved ABTS radical cation decolorization assay. Free Radic Biol Med., 1999; 26(9-10): 1231-1237.

15. Suciu M, Suciu L, Vlaia L, Voicu M, Buda V, Drăgan L, Andor M, Vlaia V, Cristescu C, Prevalence and the patterns of use of non-steroidal anti-inflammatory drugs (NSAIDs) and paracetamol among the general population. Farmacia, 2019; 67(2): 337-345.

16. Tabassum S, Kumara THS, Jasinski JP, Millikan SP, Yathirajan HS, Ganapathy PSS, Sowmya HBV, More SS, Nagendrappa G, Kaur M, Jose G, Synthesis, crystal structure, ABTS radical-scavenging activity, antimicrobial and docking studies of some novel quinoline derivatives. J Mol Struct., 2014; 1070: 10-20.

17. Wolszleger (Drăgan) M, Stan CD, Panzariu A, Jitareanu A, Profire L, New thiazolidine-4-ones of ferulic acid with antioxidant potential. Farmacia, 2015; 63(1): $150-154$. 